\title{
The association between metabolic syndrome and sleep symptoms and sleep hygiene in the elderly in Northern Taiwan
}

\author{
Meng-Ting Tsou ${ }^{1}$ \\ ${ }^{1}$ Family Medicine, Mackay Memorial Hospital, Taipei, Taiwan; mttsou@gmail.com
}

Received 1 October 2013; revised 1 November 2013; accepted 8 November 2013

Copyright (C) 2014 Meng-Ting Tsou. This is an open access article distributed under the Creative Commons Attribution License, which permits unrestricted use, distribution, and reproduction in any medium, provided the original work is properly cited. In accordance of the Creative Commons Attribution License all Copyrights @ 2014 are reserved for SCIRP and the owner of the intellectual property Meng-Ting Tsou. All Copyright (c) 2014 are guarded by law and by SCIRP as a guardian.

\section{ABSTRACT}

Background: Sleep symptoms can predict the development of metabolic syndrome (MetS) in the general population. This study focused on the association between MetS and commonly reported sleep symptoms and personal sleep hygiene habits. The goal of this study was to help individuals to make healthier decisions and thereby prevent MetS. Methods: A cross-sectional survey was conducted among elderly people (age $\geq 65$ years) who underwent a senior citizen health examination between March and November 2009. A total of 1181 participants (433 men, 36.7\%; 748 women, $63.3 \%$ ) were surveyed. MetS was defined using the modified Adult Treatment Panel III (ATP III). The participants completed a sleep questionnaire and a MetS evaluation. Multiple linear regression analysis was used to examine the relationship of the number of MetS components with individual sleep symptoms and sleep hygiene. Results: The specific symptom of insomnia (difficulty falling asleep [DFA]) increased the number of MetS risk factors significantly (coefficient $=0.252, p=0.003, R^{2}=$ $0.8)$. The duration of insomnia was not associated with the risk of MetS. Sleep hygiene habits, including taking a nap lasting longer than 1 hour during the day (coefficient $=0.256, p=$ $0.001, R^{2}=0.7$, drinking caffeine-containing drinks during the day (coefficient $=0.233, p=$ $0.013, R^{2}=0.5$ ), and getting regular exercise each day (coefficient $=-0.179, p=0.024, R^{2}=$ $0.4)$, affected the number of MetS risk factors. Conclusion: DFA and unhealthy personal sleep hygiene habits increased the number of MetS risk factors in the community-dwelling elderly population in northern Taiwan. We propose that early evaluation of sleep symptoms and sleep hygiene can help identify individuals at risk for MetS, and early intervention would result in lower occurrence rates of MetS in elderly Taiwanese.

\section{KEYWORDS}

Metabolic Syndrome; Sleep Symptoms; Sleep Hygiene; Risk Factor; Elderly Taiwanese

\section{INTRODUCTION}

The Elderly Nutritional and Health Survey in Taiwan (NAHSIT-II, 1999-2000, N = 2432 [1,2]; NAHSIT 20052008, $N=897$ [3]), a national survey of non-institutionalized elderly Taiwanese $(65$ years of age), showed that in the period between 1999 and 2008, the prevalence of metabolic syndrome (MetS) increased progressively from $25.5 \%$ to $44.5 \%$ in elderly men and from $46.8 \%$ to $57.3 \%$ in elderly women [1-3]. Therefore, identifying the modifiable risk factors associated with the development of MetS is of critical public health importance.

Several prospective studies have documented an independent relationship between sleep disturbances and an increased risk of developing chronic diseases, including obesity, hypertension, glucose intolerance, diabetes, and MetS [4-10]. One study published in 2010 mentioned that sleep problems are not just an annoyance but have potential major public health ramifications [10]. A handful of cross-sectional studies have shown that a broader range of self-reported sleep disturbances, including difficulty initiating and maintaining sleep and poor sleep quality, as well as polysomnographically assessed sleep 
architecture, are associated with the prevalence of MetS [11,12].

Sleep is impaired by certain unhealthy habits. Early detection and treatment of insomnia is important for both the management and prevention of these comorbid disorders [13]. For better management of insomnia, sleep hygiene education should be provided first [13]. Unhealthy sleep habits include consumption of caffeine or sympathomimetic or other stimulant drugs, exercise or excitement late in the evening, and an irregular sleep-wake schedule. Insomniacs should adhere to a regular awakening time and avoid naps regardless of the amount of nocturnal sleep they get $[14,15]$. Good sleep hygiene can improve sleep and avoid insomnia [16]. In this study, we used the questionnaire with a broader range of self-reported sleep disturbances and personal sleep hygiene habits to investigate the relationship between MetS risk factors and sleep symptoms and sleep hygiene in the elderly in northern Taiwan.

We believe the results of this study should be taken seriously by healthcare professionals, and that common sleep complaints or sleep habits should be investigated when assessing an elderly patient. Effective detection and treatment of insomnia may improve the quality of life of many patients and help them avoid the risk of MetS, even in the elderly population.

\section{METHODS}

\subsection{Study Population}

This study targeted elderly individuals (age $\geq 65$ years) who underwent a health examination between March and November 2009 at one of several medical centers in Taipei City. Data were collected using a questionnaire administered by the interviewer in a face-to-face session in order to avoid write-in errors. A total of 1799 elderly individuals underwent physical examinations in the hospitals. After excluding 420 individuals who provided incomplete questionnaires, data from a total of 1379 individuals were included in our study for data analysis. However, due to incomplete data on the 5 MetS risk factors, another 198 cases were eliminated. Thus, complete data from a total of 1181 individuals (recovery rate, 85.6\%) were used for the eventual analysis. All subjects provided written informed consent. Our study was approved by the Institutional Review Board of our hospital and issued research project number 09MMHISO11.

\subsection{Data Collection}

During the participants' examination at our facility, a 12-hour fasting blood draw was performed, and sitting blood pressure (BP) and anthropometric measurements were made. After the participant had sat and rested for 5 - $10 \mathrm{~min}$, BP readings were taken twice in the right arm, were taken 30 seconds apart. If these 2 BP readings differed by more than $10 \mathrm{mmHg}$, a third BP measurement was made. The average of the 2 closest readings was calculated and used in our analysis [17]. Waist circumference was measured at the standard point at the end of a relaxed expiration with a tape measure positioned parallel to the floor and with participants standing with arms akimbo [18]. NaF plasma was collected for analysis of fasting plasma glucose (FPG), and serum was collected for lipid measurements. Fasting total cholesterol, triglyceride (TG), and FPG levels were measured by an automated system (Vitros 550/750; Ortho-Clinical Diagnostics Inc., Rochester, NY, USA). Electrophoresis was performed to measure high-density lipoprotein cholesterol (HDL-C).

\subsection{Diagnostic Criteria for MetS}

The modified NCEP-ATPIII criteria were used in this study. MetS was defined by the presence of any 3 of the following 5 conditions: abdominal obesity (AO; waist circumference, $\geq 90 \mathrm{~cm}$ for men and $\geq 80 \mathrm{~cm}$ for women), hypertriglyceridemia (HTG; TG, $\geq 150 \mathrm{mg} / \mathrm{dl}$ ), low serum HDL-C level (HDL-C, $\leq 40 \mathrm{mg} / \mathrm{dl}$ for men and $\leq 50 \mathrm{mg} / \mathrm{dl}$ for women), hypertension (HT; systolic BP, $\geq 130$, diastolic $\mathrm{BP}, \geq 85 \mathrm{mmHg}$, or the use of antihypertensive agents), and hyperglycemia (HG; FPG, $\geq 100 \mathrm{mg} / \mathrm{dl}$ or the use of antihyperglycemic agents) [19].

\subsection{Sleep Symptoms/Insomnia Syndrome}

The Chinese version of the Athens Insomnia Scale (CAIS) is a self-reporting instrument designed to screen for insomnia symptoms, with the diagnostic criterion for primary insomnia being a frequency of $\geq 3$ times per week over the preceding month. CAIS-5 demonstrated that items 1 to 5 of AIS (nighttime symptoms) are useful for screening and diagnosing insomnia in clinical practice, and have satisfactory reliability and validity. The Cronbach's $\alpha$ of internal consistency for these assessments reached $0.82-0.84$, and correlation coefficients of test-retest reliability were $0.84-0.86$. The correlation coefficients between the CAIS and Insomnia Self-assessment Inventory (ISAI) were 0.72 - 0.76. The suggested cut-off points for insomnia in this ethnic Chinese population are 5 for the CAIS-5 (area under curve, AUC $=0.90$, $\mathrm{p}<0.01)$ [20].

The sleep symptoms assessed included at least 1 insomnia-related sleep complaint (difficulty falling asleep, difficulty staying asleep, feeling that sleep is not refreshing, or frequent awakening from sleep or dreaming), with a frequency of $\geq 3$ times per week. Insomnia duration was divided into 5 groups: less than 1 month, 1 month to $1 / 2$ year, $1 / 2$ year to 1 year, 1 year to 3 years, and more than 3 years. 


\subsection{Sleep Hygiene}

In previous studies, the following steps were suggested to improve sleep hygiene and both the quality and quantity of sleep, including habits that promote sleep: going to bed at the same time each day, getting regular exercise every day, drinking milk to assist in falling asleep, using the bed only for sleep and sex. They also included avoiding habits that interfere with sleep such as napping for more than 1 hour during the day, staying in bed when awake, frequently going to the toilet in the middle of the night, becoming anxious easily, being depressed, taking worries to bed, using alcohol as a sleep aid, smoking before sleep, and drinking beverages containing caffeine during the day $[14,15]$.

\subsection{Covariates}

Detailed demographic histories, including gender, education level, and living conditions, were taken during the visit. Education level was classified to one of the following 5 levels: illiterate, elementary school, junior high school, senior high school, and college or higher; living conditions were defined as "single" or "living with family".

In addition, lifestyle characteristics, including smoking history (current or former smokers versus individuals who had never smoked) and alcohol consumption ( $>4$ drinks per week versus $\leq 4$ drinks per week) were measured by study-specific questionnaires. Physical activity was assessed by the Lipids Research Clinics Questionnaire and analyzed as a categorical variable (sedentary versus non-sedentary lifestyle) [21].

\subsection{Statistical Methods}

SAS 9.0 software (SAS Inc., Cary, NC, USA) and SPSS 17.0 (SPSS Inc., Chicago, IL, USA) software were used for statistical analysis. Continuous variables were reported in terms of mean \pm standard deviation (SD) values. Difference in the number of MetS risk factors was compared between those who had sleep symptoms and unhealthy sleep hygiene versus those who did not have these symptoms and habits by using the ANOVA and independent-sample T test. Multivariate linear regression was used to examine the relationship between individual sleep disorder symptoms (model 1), sleep hygiene issues (model 2), or both (model 3), and the number of MetS risk factors, with adjustment for covariates including age, gender, living status (with family/single), smoking status (ever/never), alcohol consumption (0 - 3 drinks per week/4 or more drinks per week), sedentary lifestyle (yes/no), and education level (non-illiterate/ illiterate).

\section{RESULTS}

In our study, $43.5 \%$ of the participants $(n=1181)$ met the criteria of insomnia syndrome. As shown in Table 1, the incidence of insomnia syndrome among women was higher than that among men ( $\mathrm{p}<0.01)$; the mean age of the insomniac participants was lower than that of the non-insomniac participants $(\mathrm{p}<0.01)$. The other factors, including smoking status, alcohol consumption, sedentary lifestyle, and education level showed no significant difference. Single living status was more frequently noted among participants with insomnia $(\mathrm{p}=0.01)$. Table 2 shows a comparison of the number of MetS risk factors according to the presence of the insomnia syndrome, insomnia duration, and the presence of sleep symptoms. Of the sleep symptoms, only participants with difficulty falling asleep (DFA) had a higher number of MetS risk factors than non-DFA participants ( $p=0.007$ ). Other sleep symptom variables were not related to the number of MetS risk factors.

In Table 3, the number of MetS risk factors is compared according to sleep hygiene habits. The results showed that napping for more than 1 hour in the daytime, using alcohol as a sleep aid, smoking before sleep, and drinking caffeine-containing drinks during the day increased the number of MetS risk factors $(p<0.05)$.

In the multivariate linear regression models (Table 4), which included each sleep symptom in model 1 (adjusted for age, gender, living status, smoking status, alcohol consumption, sedentary lifestyle, and education level), only DFA significantly increased the number of MetS risk factors (intercept: 2.024, coefficient $=0.26, \mathrm{p}<$ $0.001, R^{2}=0.7$ ). In model 2 , fourteen sleep hygiene habits were analyzed. The results showed the intercept was 2.036 ( $\mathrm{p}<0.001$ ), and adding the behavior of napping more than 1 hour in the daytime increased MetS risk factors by $0.247\left(p=0.001, R^{2}=0.7\right.$ ); the other factors included drinking caffeine-containing drinks during the day (coefficient $=0.227, \mathrm{p}=0.016, \mathrm{R}^{2}=0.5$ ), and getting regular exercise every day (coefficient $=-0.189, \mathrm{p}=$ $0.017, \mathrm{R}^{2}=0.5$ ) also increased the number of MetS risk factors. Sleep symptoms and sleep hygiene habits were analyzed in model 3, and the intercept was 1.958 (p < 0.001 ); napping for longer than 1 hour in the daytime (coefficient $=0.256, \mathrm{p}=0.001, \mathrm{R}^{2}=0.7$ ); DFA (coefficient $=0.252, \mathrm{p}=0.003, \mathrm{R}^{2}=0.8$ ); drinking caffeinecontaining drinks during the day (coefficient $=0.233, \mathrm{p}=$ $0.013, \mathrm{R}^{2}=0.5$ ), and getting regular exercise each day (coefficient $=-0.179, \mathrm{p}=0.024, \mathrm{R}^{2}=0.4$ ). The remaining insomnia symptoms and sleep hygiene habits were not related to the risk of MetS.

\section{DISCUSSION}

Insomnia is prevalent in adults, with 30 to 40 percent of adults indicating they experienced some level of insomnia within any given year, and about 10 to 15 percent indicating that the insomnia was chronic, severe, or both, 
Table 1. Characteristics of the subjects by non-insomnia and insomnia $(\mathrm{N}=1181)$.

\begin{tabular}{|c|c|c|c|}
\hline Variable & $\begin{array}{c}\text { Non-insomnia } \\
\text { (667, 56.5\%) } \\
\text { N (\%) }\end{array}$ & $\begin{array}{c}\text { Insomnia } \\
\text { (514, 43.5\%) } \\
\text { N (\%) }\end{array}$ & $\mathrm{p}$ value \\
\hline Gender & & & $<0.001^{\dagger}$ \\
\hline Male & $332(49.6)$ & $189(36.7)$ & \\
\hline Female & $335(50.4)$ & 325 (63.3) & \\
\hline Age (mean \pm SD) & $74.2 \pm 5.7$ & $73.5 \pm 5.3$ & $0.01^{\mathrm{a}^{*}}$ \\
\hline Smoking (recent 1/2 year) & & & 0.07 \\
\hline No & $632(94.7)$ & $498(96.8)$ & \\
\hline Yes & $35(5.3)$ & $16(3.2)$ & \\
\hline Alcohol (recent 1/2year) & & & 0.21 \\
\hline No & $590(88.4)$ & $466(90.6)$ & \\
\hline Yes & 77 (11.6) & $48(9.4)$ & \\
\hline Exercise (recent $1 / 2$ year) & & & 0.09 \\
\hline No & $61(9.2)$ & $62(12.1)$ & \\
\hline Yes & $606(90.8)$ & 452 (87.9) & \\
\hline Living condition & & & $0.001^{\mathrm{b} \dagger}$ \\
\hline Single & $42(6.3)$ & $60(11.6)$ & \\
\hline With family & $625(93.7)$ & $454(88.4)$ & \\
\hline Education level & & & 0.80 \\
\hline Illiterate & $35(5.3)$ & $27(5.2)$ & \\
\hline Elementary & 200 (29.9) & $157(30.6)$ & \\
\hline Junior high school & $111(16.7)$ & $92(17.9)$ & \\
\hline Senior high school & $168(25.1)$ & $134(26.1)$ & \\
\hline$\geqslant$ College & $153(23.0)$ & $104(20.2)$ & \\
\hline
\end{tabular}

Note: chi-square test, a: Student's t test and b: fisher's exact test were used, compare with non-insomnia and insomnia group. "means $\mathrm{p}<0.05,{ }^{\dagger}$ means $\mathrm{p}$ $<0.01$.

although most patients do not mention it during routine office visits [22]. The prevalence of insomnia increases with age and is more common in women [23]. Insomnia has been reported to underlie the development and aggravation of MetS, including hypertension, cardiovascular disease, and diabetes [13]. Current research suggests that getting enough sleep is important for memory consolidation, proper functioning of the immune system, metabolism, weight control, cardiovascular health, and maintaining a pleasant mood [24]. Early detection and treatment of insomnia is important for both the management and prevention of these comorbid disorders [13].

In our cross-sectional study, we found the sleep symptom of DFA increased the number of MetS risk factors in the community-dwelling elderly population in northern Taiwan $\left(\right.$ coefficient $=0.252, \mathrm{p}=0.003, \mathrm{R}^{2}=$ 0.8). A 2010 American prospective study of communitydwelling middle-aged adults evaluated sleep symptoms commonly presented in clinical practice in relation to the development of the MetS over a 3-year period, and found that DFA increased the risk of MetS by $80 \%$ [10]. This previous finding raises the possibility that associated
Table 2. Risk factors No. of MetS according to insomnia syndrome, duration, and symptoms by ANOVA, independent samples $\mathrm{T}$ test $(\mathrm{N}=1181)$.

\begin{tabular}{|c|c|c|c|}
\hline Character & N (\%) & Risk factor & $\mathrm{p}$ value \\
\hline Insomnia syndrome & & & 0.13 \\
\hline No & 667 (56.5) & $2.01 \pm 1.3$ & \\
\hline Yes & $514(43.5)$ & $2.12 \pm 1.3$ & \\
\hline Insomnia duration & & & $0.67^{\mathrm{a}}$ \\
\hline Less than 1 month & $78(6.6)$ & $2.01 \pm 1.2$ & \\
\hline 1 month - $1 / 2$ year & $52(4.4)$ & $2.14 \pm 1.4$ & \\
\hline $1 / 2$ year - 1 year & $71(6.0)$ & $2.15 \pm 1.5$ & \\
\hline 1 year - 3 years & $110(9.3)$ & $2.12 \pm 1.3$ & \\
\hline More than 3 years & $203(17.2)$ & $2.16 \pm 1.3$ & \\
\hline \multicolumn{4}{|l|}{ Sleep symptoms } \\
\hline Difficulty falling sleep & & & $0.007^{\mathrm{tb}}$ \\
\hline No & 865 (73.2) & $1.99 \pm 1.3$ & \\
\hline Yes & 316 (26.8) & $2.23 \pm 1.4$ & \\
\hline Difficulty staying sleep & & & $0.08^{\mathrm{b}}$ \\
\hline No & 977 (82.7) & $2.09 \pm 1.3$ & \\
\hline Yes & 204 (17.3) & $1.91 \pm 1.3$ & \\
\hline Non-refreshing sleep & & & $0.93^{\mathrm{b}}$ \\
\hline No & 1132 (95.9) & $2.06 \pm 1.3$ & \\
\hline Yes & $49(4.1)$ & $2.04 \pm 1.1$ & \\
\hline $\begin{array}{l}\text { Frequent awaking from } \\
\text { sleep or dreamy }\end{array}$ & & & $0.24^{\mathrm{b}}$ \\
\hline No & $1063(90.0)$ & $2.04 \pm 1.3$ & \\
\hline Yes & $118(10.0)$ & $2.19 \pm 1.2$ & \\
\hline $\begin{array}{l}\text { Combination of sleep } \\
\text { symptoms }\end{array}$ & & & $0.55^{\mathrm{a}}$ \\
\hline No & 667 (56.5) & $2.01 \pm 1.3$ & \\
\hline 1 symptom only & 368 (31.2) & $2.15 \pm 1.3$ & \\
\hline Combined 2 symptoms & $111(9.4)$ & $2.05 \pm 1.3$ & \\
\hline Combined 3 symptoms & $24(2.0)$ & $2.18 \pm 1.4$ & \\
\hline Combined 4 symptoms & $11(0.9)$ & $2.11 \pm 1.1$ & \\
\hline
\end{tabular}

Note: analysis—a: ANOVA, b: Independent samples T test. ${ }^{*}$ means $\mathrm{p}<0.05$, ${ }^{\dagger}$ means $\mathrm{p}<0.01$.

symptoms may in fact be a causal risk factor for cardiometabolic dysregulation in the general population, although fewer studies have addressed this issue in the worldwide elderly population.

For better management of insomnia, sleep hygiene education should be provided first [13]. In our study, we found that some sleep hygiene habits affected the number of MetS risk factors: napping for longer than 1 hour during the daytime (coefficient $=0.256, \mathrm{p}=0.001, \mathrm{R}^{2}=$ $0.7)$; drinking caffeine-containing drinks during the day (coefficient $=0.233, \mathrm{p}=0.013, \mathrm{R}^{2}=0.5$ ), and getting regular exercise every day (coefficient $=-0.179, \mathrm{p}=$ $0.024, R^{2}=0.4$ ). The aim of our study was to enable healthier decisions and to prevent MetS, while increasing the overall quality of life.

Previous evidence has linked DFA with the incidence 
Table 3. Risk factors No. of MetS according to sleep hygiene.

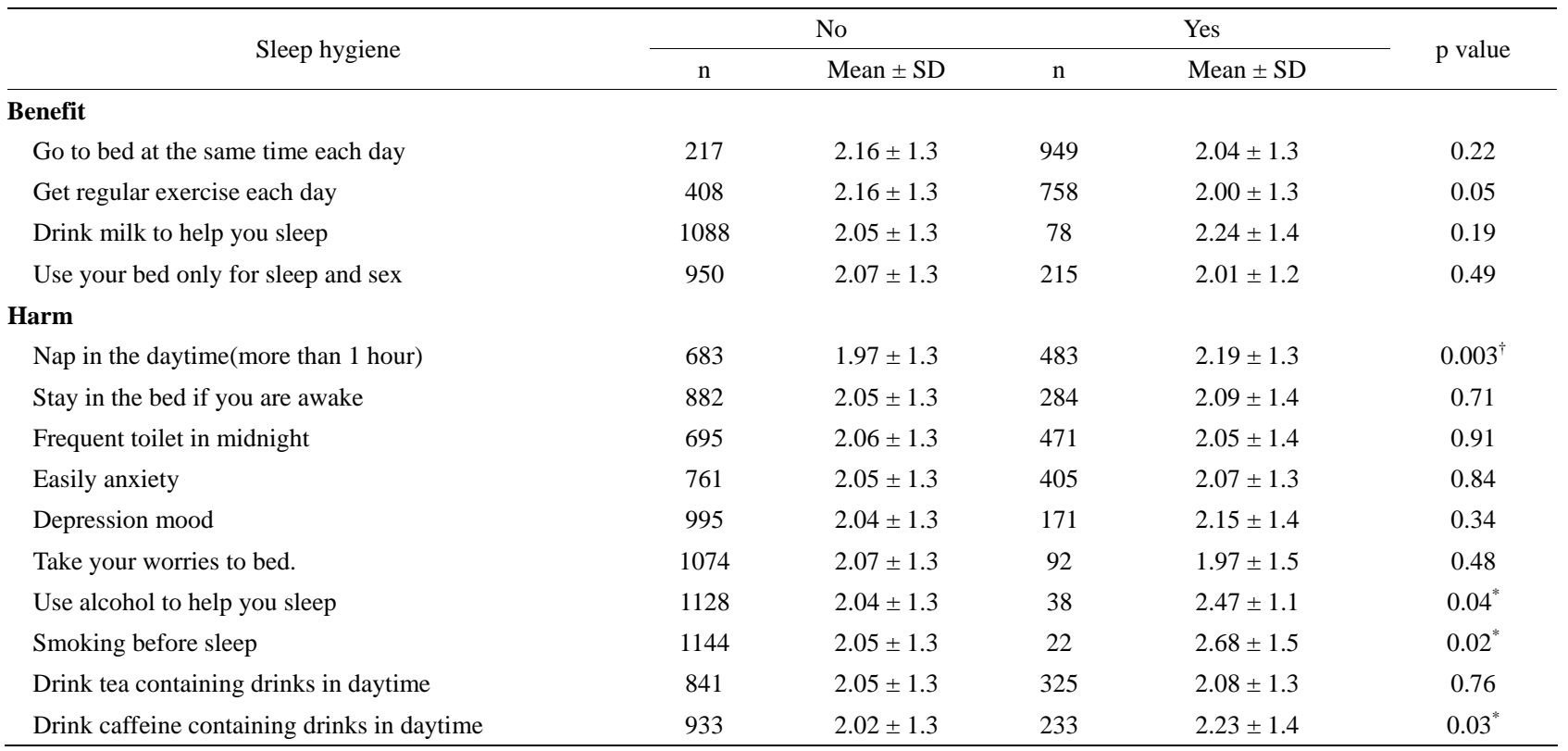

Note: Independent samples T test. ${ }^{*}$ means $\mathrm{p}<0.05,{ }^{\dagger}$ means $\mathrm{p}<0.01$.

Table 4. The relationship of insomnia symptoms, sleep hygiene and MetS factors by multiple linear regression [dependent variables: Risk factor No. of MetS; independent variables: insomnia symptoms (4) and sleep hygiene (14)].

\begin{tabular}{|c|c|c|c|c|}
\hline & \multicolumn{2}{|c|}{ No. of MetS factors } & \multirow[t]{2}{*}{$\mathrm{R}^{2}$} & \multirow[t]{2}{*}{ Accumulate $\mathrm{R}^{2}$} \\
\hline & Coefficient (95\% CI) & $\mathrm{p}$ value & & \\
\hline \multicolumn{5}{|l|}{ Model 1: Sleep symptoms } \\
\hline Intercept & $2.024(1.933-2.116)$ & $<0.001^{\dagger}$ & & \\
\hline Difficulty falling sleep (ref.: no) & $0.260(0.092-0.429)$ & $0.003^{\dagger}$ & 0.7 & 0.7 \\
\hline \multicolumn{5}{|l|}{ Model 2: Sleep hygiene } \\
\hline Intercept & $2.036(1.895-2.176)$ & $<0.001^{\dagger}$ & & \\
\hline Nap in the daytime(more than 1 hour) (ref.: no) & $0.247(0.096-0.398)$ & $0.001^{\dagger}$ & 0.7 & 0.7 \\
\hline Drink caffeine containing drinks in daytime (ref.: no) & $0.227(0.042-0.412)$ & $0.016^{*}$ & 0.5 & 1.2 \\
\hline Get regular exercise each day (ref.: no) & $-0.189(-0.345--0.033)$ & $0.017^{*}$ & 0.5 & 1.7 \\
\hline \multicolumn{5}{|l|}{ Model 3: $1+2$} \\
\hline Intercept & $1.958(1.808-2.108)$ & $<0.001^{\dagger}$ & & \\
\hline Nap in the daytime(more than 1 hour) (ref.: no) & $0.256(0.106-0.407)$ & $0.001^{\dagger}$ & 0.7 & 0.7 \\
\hline Difficulty falling sleep (ref.: no) & $0.252(0.084-0.419)$ & $0.003^{\dagger}$ & 0.8 & 1.5 \\
\hline Drink caffeine containing drinks in daytime (ref.: no) & $0.233(0.049-0.418)$ & $0.013^{*}$ & 0.5 & 2.0 \\
\hline Get regular exercise each day (ref.: no) & $-0.179(-0.335--0.023)$ & $0.024^{*}$ & 0.4 & 2.4 \\
\hline
\end{tabular}

Note: Adjustment for covariates including age, sex, living status (with family/single), smoking status (ever/never), alcohol consumption ( 0 - 3 drinks per week/4 or more drinks per week), sedentary lifestyle (yes/no), and education level (non-illiterate/illiterate). ${ }^{*}$ means $\mathrm{p}<0.05$, ${ }^{\dagger}$ means $\mathrm{p}<0.01$.

of diabetes and mortality [25]. Prolonged sleep latency may reflect a state of emotional and physiological hyperarousal, which has been linked to heightened sympathetic activation and hypercortisolemia [26] — both of which have been implicated in the pathophysiology of insulin resistance and MetS. Evidence suggests that healthy sleep habits, according to the National Sleep Foundation [27], such as avoiding napping for longer than 1 hour during the day, performing regular exercise every day, avoiding the consumption of caffeinated beverages at least 8 hours before bedtime, improve insomnia and sleep disorder symptoms, and our results are compatible with these recommendations. These habits affect the insomnia pathway via the same mechanism-heightened sympathetic activation and hypercortisolemia [26].

Study limitations include the fact that this was a crosssectional survey, as the aging population lives in several parts of the northern metropolitan area, and may not be representative of the entire elderly population. The prevalence of insomnia may be overestimated for some el- 
derly individuals who may go to a medical center to receive physical examinations on their own, because they may pay more attention to their health and insomnia than other elderly individuals. Our study provided community data about the relationship between common sleep problems and the number of MetS risk factors in the elderly population in northern Taiwan. We wanted to find out whether the influence of specific risk factors could be determined in the elderly population that has access to medical treatment, because these issues would therefore warrant early diagnosis and intervention.

This study provides evidence that supports the relationship between the number of MetS risk factors and commonly reported sleep disorder symptoms such as DFA and sleep hygiene habits such as napping for longer than 1 hour during the daytime, drinking caffeine-containing drinks during the day, and getting regular exercise every day. Given these results in the general elderly population, and that sleep complaints and unhealthy sleep hygiene are considerably more prevalent than either the insomnia syndrome or other personal habits, these findings have far-reaching implications for public health, particularly given the epidemic levels of obesity and its associated cardiometabolic consequences, which are associated with sleep disturbances. Our findings reflect the utility of investigating common sleep complaints and personal sleep hygiene issues in routine clinical practice as these individuals may be at elevated risk for the development of MetS [28].

\section{REFERENCES}

[1] Tsai, H.J. and Tsai, A.C. (2012) Prevalence of metabolic syndrome in elderly Taiwanese: Results from the nutrition and health survey in Taiwan (1999-2000). http://apha.confex.com/apha/135am/techprogram/paper_1 50632.htm

[2] Lee, L.T., Chang, Y.C., Huang, K.C., Pan, W.H. and Chen, C.Y. (2005) The prevalence survey of metabolic syndrome of the elderly in Taiwan. Taiwan Journal of Clinical Gerontology and Geriatrics, 1, 1-18.

[3] (2012) 2005-2008 Nutrition and health survey in Taiwan (NAHSIT). http://nahsit.nhri.org.tw/node/14

[4] Reichmuth, K.J., Austin, D., Skatrud, J.B. and Young, T. (2005) Association of sleep apnea and type 2 diabetes: A population-based study. American Journal of Respiratory and Critical Care Medicine, 172, 1590-1595. http://dx.doi.org/10.1164/rccm.200504-637OC

[5] Ayas, N.T., White, D.P., Al-Delaimy, W.K., Manson, J.E., Stampfer, M.J., Speizer, F.E., Patel, S. and Hu, F.B. (2003) A prospective study of self-reported sleep duration and incident diabetes in women. Diabetes Care, 26, 380-384. http://dx.doi.org/10.2337/diacare.26.2.380

[6] Gangswich, J.E., Heymsfield, S.B., Boden-Albala, B., Buijs, R.M., Kreier, F., Pickering, T.G., Rundle, A.G., Zammit, G.K. and Malaspina, D. (2007) Sleep duration as a risk factor for diabetes incidence in a large US sample. Sleep, 30, 1667-1673.

[7] Kawakami, N., Takatsuka, N. and Schimizu, H. (2004) Sleep disturbance and onset of type 2 diabetes. Diabetes Care, 27, 282-283. http://dx.doi.org/10.2337/diacare.27.1.282

[8] Nilsson, P.M., Roost, M., Engstrom, G., Hedblad, B. and Berglund, G. (2004) Incidence of diabetes in middle-aged men is related to sleep disturbances. Diabetes Care, 27, 2464-2469. http://dx.doi.org/10.2337/diacare.27.10.2464

[9] O’Connor, G.T., Caffo, B., Newman, A.B., Quan, S.F., Rapoport, D.M., Redline, S., Resnick, H.E., Samet, J. and Shahar, E. (2009) Prospective study of sleep-disordered breathing and hypertension: The sleep heart health study. American Journal of Respiratory and Critical Care Medicine, 179, 1159-1164. http://dx.doi.org/10.1164/rccm.200712-18090C

[10] Troxel, W.M., Buysse, D.J., Matthews, K.A., Kip, K.E., Strollo, P.J., Hall, M., Drumheller, O. and Reis, S.E. (2010) Sleep symptoms predict the development of the metabolic syndrome. Sleep, 33, 1633-1640.

[11] Hall, M.H., Muldoon, M.F., Jennings, J.R., Buysse, D.J., Flory, J.D. and Manuck, S.B. (2008) Self-reported sleep duration is associated with the metabolic syndrome in midlife adults. Sleep, 31, 635-643.

[12] Jennings, J.R., Muldoon, M.F., Hall, M., Buysse, D.J. and Manuck, S.B. (2007) Self-reported sleep quality is associated with the metabolic syndrome. Sleep, 30, 219-223.

[13] Takaesu, Y. and Inoue, Y. (2012) Treatment strategy of insomnia for the patients with metabolic syndrome. $\mathrm{Ni}$ hon Rinsho, 70, 1216-1221.

[14] University of Maryland Medical Center (2010) Sleep Disorders Center-Sleep hygiene: Helpful hints to help you sleep. http://www.umm.edu/sleep/sleep hyg.htm

[15] (2012) Sleep and wakefulness disorders: Sleep hygiene. http://www.merckmanuals.com/professional/neurologic d isorders/sleep and wakefulness disorders/approach to the_patient_with_a_sleep_or_wakefulness_disorder.html \#v1039077

[16] (2010) Insomnia and excessive daytime sleepiness (EDS). http://www.merckmanuals.com/professional/neurologic_d isorders/sleep_and_wakefulness_disorders/insomnia_and _excessive_daytime_sleepiness_eds.html

[17] Hwang, L.C., Bai, C.H. and Chen, C.J. (2006) Prevalence of obesity and metabolic syndrome in Taiwan. Journal of the Formosan Medical Association, 105, 626-635. http://dx.doi.org/10.1016/S0929-6646(09)60161-3

[18] Bureau of Health Promotion, Department of Health (Taiwan) (2012) The method of measuring waist circumference of adult. http://www.health99.doh.gov.tw/TXT/PreciousLifeZone/ print.aspx?TopIcNo=52\&DS=1-Article

[19] Bureau of Health Promotion Database (2014) The criteria of metabolic syndrome. http://www.youth.com.tw/db/epaper/es002001/eb2403.htm

[20] A Validation Study of the Chinese Version of the Athens Insomnia Scale. Taiwanese Journal of Psychiatry, 3, 4352. 
[21] Ainsworth, B.E., Jacobs, D.R. and Leon, A.S. (1993) Validity and reliability of self-reported physical activity status: The Lipid Research Clinics Questionnaire. Medicine \& Science in Sports \& Exercise, 25, 92-98. http://dx.doi.org/10.1249/00005768-199301000-00013

[22] Insomnia: Assessment and management in primary care. National heart, lung, and blood institute working group on insomnia, Bethesda, Maryland. (1999) American Family Physician, 59, 3029-3038.

[23] Foley, D.J., Monjan, A.A., Brown, S.L., Simonsick, E.M., Wallace, R.B. and Blazer, D.G. (1995) Sleep complaints among elderly persons: An epidemiologic study of three communities. Sleep, 18, 425-432.

[24] Harvard Health Publications (2011) Importance of sleep: Six reasons not to scrimp on sleep. Harvard University. http://www.health.harvard.edu/press_releases/importance of sleep and health

[25] Dew, M.A., Hoch, C.C., Buysse, D.J., Monk, T.H., Begley, A.E., Houck, P.R., Hall, M., Kupfer, D.J. and Rey- nolds, C.F. (2003) Healthy older adults' sleep predicts allcause mortality at 4 to 19 years of follow-up. Psychosomatic Medicine, 65, 63-73. http://dx.doi.org/10.1097/01.PSY.0000039756.23250.7C

[26] Vgontzas, A.N., Chrousos, G.P., Vgontzas, A.N. and Chrousos, G.P. (2002) Sleep, the hypothalamic-pituitaryadrenal axis, and cytokines: Multiple interactions and disturbances in sleep disorders. Endocrinology and Metabolism Clinics of North America, 31, 15-36. http://dx.doi.org/10.1016/S0889-8529(01)00005-6

[27] Centers for Disease Control and Prevention (CDC) Features (2011) Sleep and sleep disorders. CDC. http://www.cdc.gov/features/sleep/

[28] Wroble, R., Nagle, B.A., Cataldi, L.A. and Monane, M. (2000) Insomnia in the elderly: Assessment and management in a primary care setting. Journal of Clinical Outcomes Management, 7, 50-58. 